\title{
Inertial navigation system based on national electronic components
}

\author{
Leonid Chelyshev ${ }^{1, *}$ \\ ${ }^{1}$ Joint Stock Company "NPO Angstrem”, 124498, Moscow
}

\begin{abstract}
The article describes the need and aspects of creating an inertial positioning system on the Russian component base. Description graphic material and elemental support with the report of its use within the project are given.
\end{abstract}

In the modern world, the issue of noise immunity and autonomy of systems, if not paramount, surely ranks high in importance. Lack of opportunities to influence a working system from the outside is a priority for engineers who design it. An inertial system makes it possible, in the absence of signals from the outside (Glonass, GPS), to understand its whereabouts with a certain margin error. [1] An inertial system is an autonomous system that provides information about the position in space by registering the inertia of the attached body.

This navigation method is necessary as part of the suppression or loss of signals because that operation only requires physical changes that are not affected by interference.

Before the start of our work, the task of creating an inertial system on the domestic component base was set. As a result of studying the technical specifications, the component base was chosen, which became the basis of the project. [2]

This list includes the following:

1. KR142EN24A as a voltage converter. According to GOST R 54073-2010, the required power for the system is $27 \mathrm{~V}$, with possible voltage drops for category I equipment. This module must convert the supply voltage on board to the voltage required to power the system $(+5 \mathrm{~V},+3.3 \mathrm{~V})$.

2. As the event handler (microcontroller) for the prototype, an STMicroelectronics product was chosen, namely STM32F103C8T6, which has proven itself in previous works, in addition to being an optimal solution in terms of price/quality ratio. Because of the specifics of the Terms of Reference, namely, the requirement of using domestic-produced electronic components, the NIIET-manufactured K1921VK01T microcontroller was chosen, which is a more potent analog of the mentioned STM. The microcontroller provides fast processing of incoming signals and quick interaction with external systems. Two possible interfaces are used to communicate with the subsystem: RS-232 and USART. Using USART, the microcontroller receives a program for its operation, and through RS-232, it

* Corresponding author: leonid.chelyshev@live.ru 
interacts with the external system. The microcontroller is equipped with capacitors, which prevent it from disconnecting from the power supply in case of voltage drop. The prototype also uses a USB interface.

Microcontroller managed LEDs notify about the readiness of the system, as well as its status.

3. Understanding the position relative to the Earth's poles requires the presence of a magnetometer in the system. In the work, we used a PMP-AMR-HX B2.1 singleaxis magnetometer, which is a complete analog of the HMC1001 (in the prototype). Since the system needs full orientation in space, the board has 3 elements described above. In addition to receiving signals by hardware, a recalibration circuit is implemented. Recalibration can be done during the operation of the system.

4. To measure accelerations, an APLU25 analog accelerometer is used, ADXL326 in the prototype. The use of an analog accelerometer has become a necessity due to the absence of an internal MEMS accelerometer. Interference is cut off from the accelerometer with the help of capacitors, and the accelerometer's software outputs an average result of work, reducing the influence of fluctuations.

5. Besides, the circuit uses an analog temperature sensor K1019EM1, which allows having an idea of temperature limit values. The system has a different minimum and maximum thermal state and therefore requires precise temperature control of the system and its members. On the board, the thermoregulation system takes care of this, which consists of a temperature sensor, a Peltier control circuit, Peltier elements ( $\mathrm{x} 4$ in the prototype). The temperature sensor records and transmits temperature readings to the microcontroller, the microcontroller then crunches the numbers and, if necessary, turns on/off the heating/cooling system. It provides a vast range of possible system temperatures.

The circuit in Fig. 1 is a prototype of the project.

The boards measure $100 \times 100 \mathrm{~mm}, 35 \times 31 \mathrm{~mm}$, are made to class 4 accuracy, which makes them relatively cheap to mass-produce. The circuit is conditionally divided into 6 parts:

1. Microcontroller and its components.

2. Voltage control circuits.

3. Power management circuits.

4. Accelerometer and its components.

5. Magnetometer circuits.

6. Circuits for external system interaction.

At the moment, the prototype is in the testing stage. The diagram in Fig. 2 represents the third axis of the ADXL magnetometer.

Also, Figure 3 shows a 3D view of the resulting microboard. The prototype board is currently undergoing comprehensive tests to understand its limit performance characteristics, as well as the accuracy of the functioning of its parts. For the system to operate stably, it is necessary to place it in a damage-resistant enclosure and calibrate it before using, to adjust magnetometer readings. The prototype uses an ADC-12 aluminum alloy housing B013MFBK.

The work resulted in a prototype of the inertial system circuit based on internal electronic components being created, modeled, and designed. The system has shown its functional capability and is currently being tested.

In the future, this device can be used in navigation systems of various vehicles. 


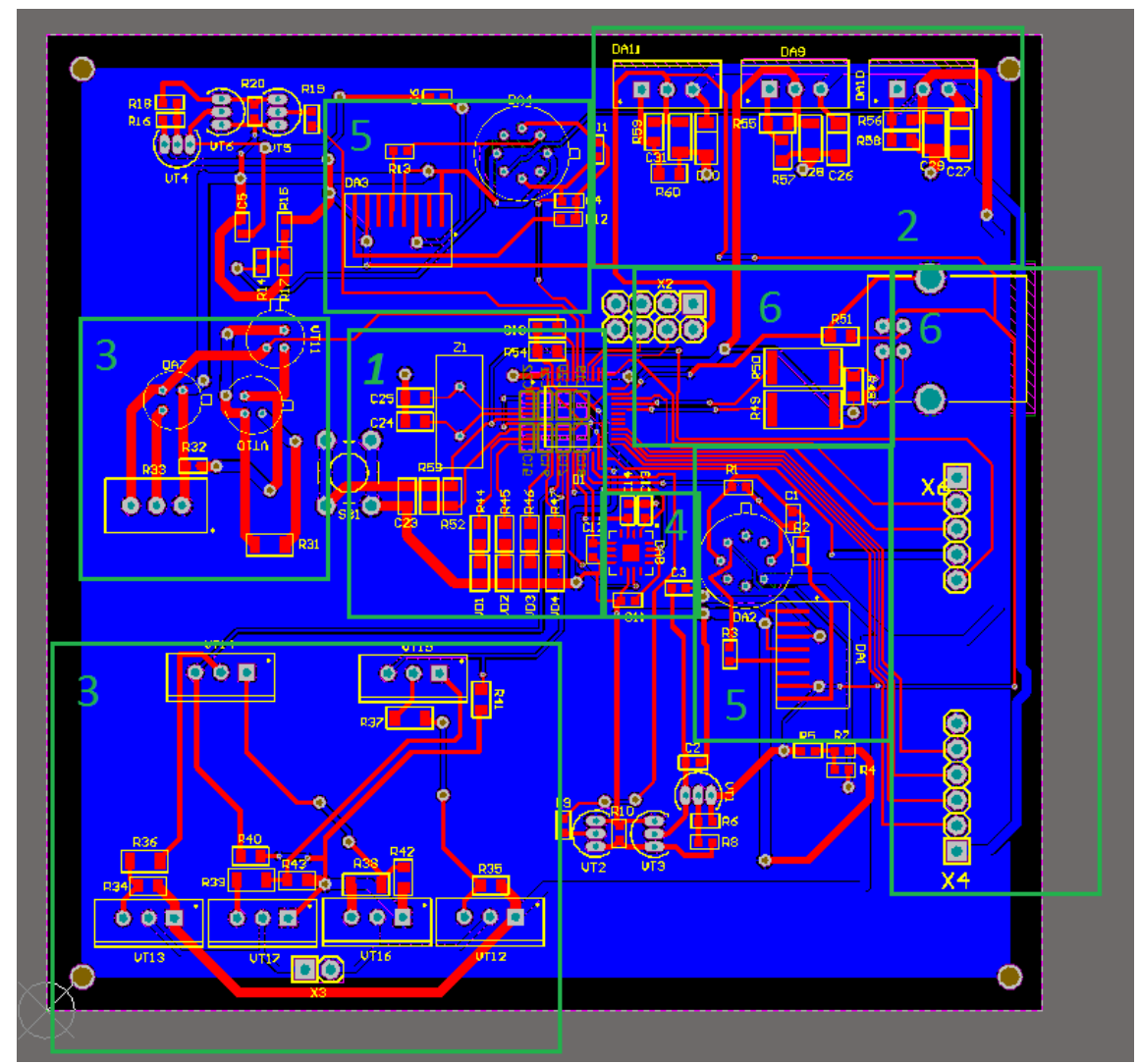

Fig. 1. Wiring diagram of the prototype inertial positioning system.

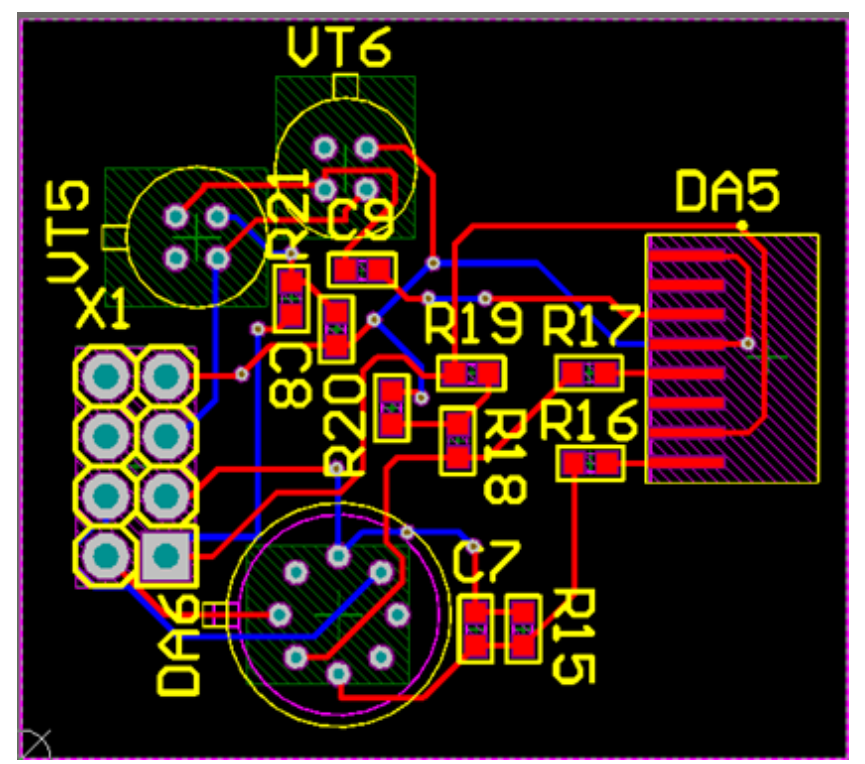

Fig. 2. Circuit board of the prototype of the third axis of the magnetometer for the inertial positioning system. 


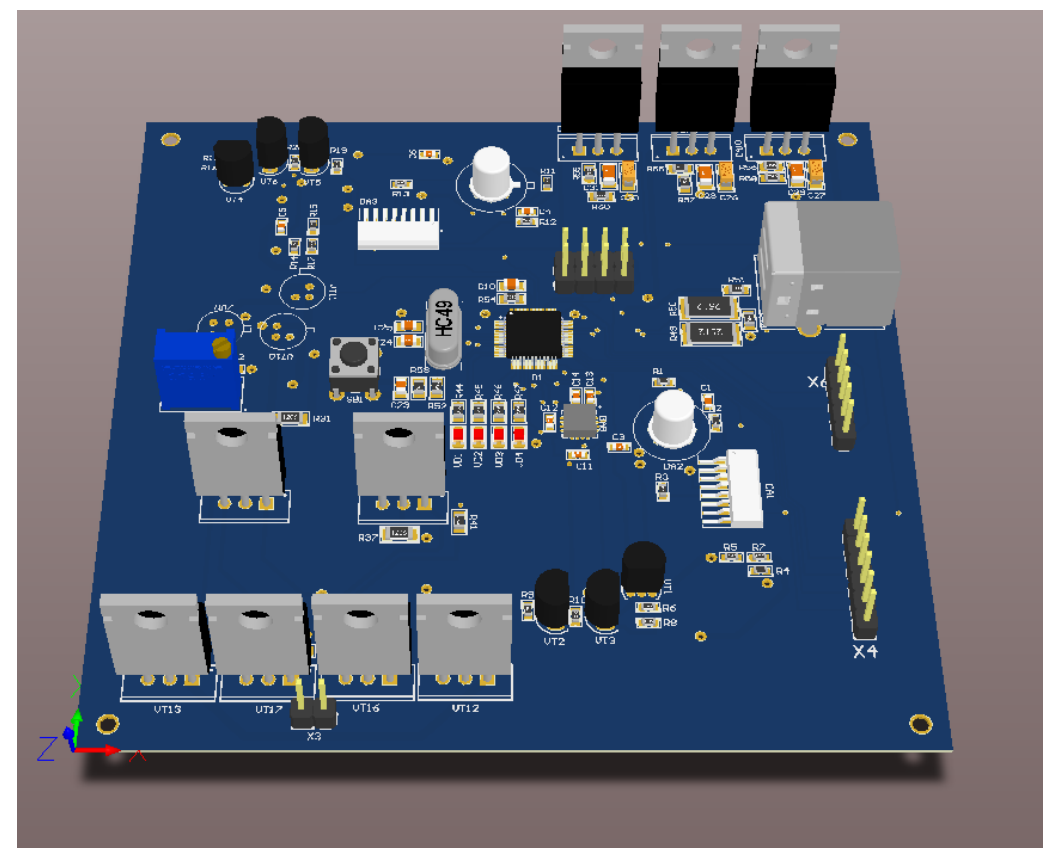

Fig. 3. A 3D view of the inertial navigation system

\section{References}

1. Matveev V.V. Investigation of errors in the strapdown inertial navigation system of a highly dynamic aircraft, Izvestiya TulGU. Technical science. No. 12-1 (2012)

2. Horowitz P., Hill. W., The Art of Circuit Engineering, (Binomial. Moscow. 706 p., 2014) 